\title{
A video programme plus a booklet was more effective than a booklet alone for increasing patient knowledge about lumbar spine treatment options for low back pain
}

\author{
Phelan EA, Deyo RA, Cherkin DC, et al. Helping patients decide about back surgery: a randomized trial of an interactive \\ video program. Spine 2001 Jan 15;26:206-12.

\section{QUESTION: Is an interactive video programme plus a booklet more effective than a booklet alone for increasing the knowledge necessary to make informed choices about surgical compared with non-surgical lumbar spine treatment for low back pain?}

\begin{abstract}
Sources of funding: The Agency for Health Care Policy and Research and the Foundation for Informed Medical Decision Making.

For correspondence: Dr E A Phelan,

Medicine/Gerontology

and Geriatric Medicine,

Harborview Medical

Center, 325 9th Avenue,

Box 359755, Seattle, WA

98104-2499, USA. Fax

$+12067318527$.
\end{abstract}

\section{Design}

Randomised (allocation concealed), unblinded, controlled trial with follow up between 27 and 56 days.

\section{Setting}

An academic, orthopaedic surgical practice in Iowa, USA.

\section{Patients}

100 patients $\geqslant 20$ years of age (mean age $50 \mathrm{y}, 56 \%$ men, $59 \%$ with a diagnosis of herniated disc) who were candidates for lumbar spine surgery and who had received non-surgical treatment for $\geqslant 4$ weeks. Exclusion criteria were emergent indication for surgery, no indication of a

\section{COMMENTARY}

With shorter hospital stays and less clinic and home visit time, nurses must find new ways to educate patients. The systematic review by O'Connor $e t$ al pointed to the importance of decision aids (written, audio, and video teaching materials) for enhancing patient knowledge and decision making. ${ }^{1}$ This study by Phelan at al provides evidence that decision aids using newer technology, an interactive video, can be successful in improving patient knowledge about lumbar spine treatments.

Confidence in the findings by Phelan at al is enhanced because of the randomised design and because the sample was large enough to show differences in postintervention patient knowledge. The authors were appropriately cautious when advising against generalising findings from this entirely white sample to other racial groups. With this caveat in mind, clinicians should note that the older, less educated patients in the sample had less baseline knowledge. Also, these patients benefited the most from the interactive videodisc plus booklet intervention. These findings can help nurses to decide who should be targeted when using videodisc and booklet type materials.

In addition to determining who will benefit, clinicians can use these findings to inform what and how we teach. Unlike the booklet, the videodisc included interviews with patients who described the positive and negative outcomes of different treatments. Including patients' perceptions of health care in teaching materials is well supported and might have contributed to the success of the videodisc intervention. ${ }^{2}$ Moreover, the videodisc intervention allowed patients to choose condition and age specific information, and patients could use menu options to stop, repeat, or explore content in greater depth. Nurses practising in settings where there is little time to individualise care could consider using interactive video aids as a method of personalising their teaching.

Finally, validity of the knowledge test used to evaluate learning was established by comparing scores of orthopaedic nurses with those of uninstructed patients. Also, Phelan had patients rate how easy teaching materials were to understand and the amount, balance, and usefulness of information. These techniques are worth nurses' consideration as they design and evaluate educational materials.

Laurel E Radwin, RN, CS, PhD Assistant Professor Department of Adult and Gerontological Nursing, College of Nursing University of Massachusetts, Boston, Massachusetts, USA

1 O'Connor AM, Rostom A, Fiset V, et al. Decision aids for patients facing health treatment or screening decisions: systematic review. BMJ 1999;319:731-4.

2 Christmas N, Kirchhoff K, Oakley M. Preparatory sensory information. In: Bulechek G, McCloskey J, editors. Nursing interventions: effective nursing treatments. Third edition. Philadelphia: WB Saunders, 1999:398-408.

surgically correctable lesion, previous low back surgery, pregnancy, cancer or infection causing back pain, severe hearing or visual impairment, unfamiliarity with English, inaccessibility because of a planned move, or lack of a home telephone. Follow up was $90 \%$.

\section{Intervention}

47 patients were allocated to receive a booklet plus the "shared decision making" videodisc (produced by the Foundation for Informed Medical Decision Making, Hanover, New Hampshire, USA) and 53 patients were allocated to receive the booklet alone.

The videodisc included computer graphics of spinal anatomy; information on low back and leg pain, and surgical and non-surgical care; and patient interviews with the use of an interactive screen that enabled patients to obtain information tailored to their age and specific causative diagnosis. Viewing occurred in an office setting during the week before the second visit with the orthopaedic surgeon. All patients received the booklet, which contained anatomic illustrations of the lumbar spine and a complete discussion of surgical and non-surgical treatment options.

\section{Main outcome measure}

Knowledge was measured by a knowledge test (scores ranging between 0 and 17) at baseline (pretest) and at 27 to 56 days (post-test).

\section{Main results}

Analysis was by intention to treat. After adjusting for baseline knowledge score, age, education, sex, and diagnosis, patients who received the videodisc plus the booklet showed a greater improvement in knowledge $(\mathrm{p}=0.05)$ but no difference in preference for surgery $(23 \% v 42 \%, \mathrm{p}=0.4)$ than those who received the booklet alone. Subgroup analyses showed that greater mean improvements in knowledge test scores were seen with the video programme plus the booklet than the booklet alone among those with low baseline knowledge scores (range 0 to 5) $(8.8 v 5.5, \mathrm{p}=0.02)$ and among those with a diagnosis of herniated disc $(\mathrm{p}=0.03)$. Among patients with medium baseline knowledge scores (range 6 to 11) and high baseline knowledge scores (range 12 to 17), no differences existed between the videodisc plus the booklet and the booklet alone for knowledge improvement.

\section{Conclusion}

A video programme plus a booklet was more effective than a booklet alone for increasing the knowledge necessary for patients to make informed choices about surgical compared with non-surgical lumbar spine treatment for low back pain. 